\title{
Promoting and Protecting Public Health: How the European Union Pharmacovigilance System Works
}

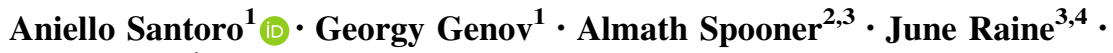 \\ Peter Arlett ${ }^{1}$
}

Published online: 22 July 2017

(C) The Author(s) 2017. This article is an open access publication

\begin{abstract}
This article provides an overview of the European Union pharmacovigilance system resulting from the rationalisation and strengthening delivered through the implementation of the revised pharmacovigilance legislation. It outlines the system aims, underlying principles, components and drivers for future change. At its core, the Pharmacovigilance Risk Assessment Committee is responsible for assessing all aspects of the risk management of medicinal products, thus ensuring that medicines approved for the European Union market are optimally used by maximising their benefits and minimising risks. The main objectives of the system are to promote and protect public health by supporting the availability of medicines including those that fulfil previously unmet medical needs, and reducing the burden of adverse drug reactions. These are achieved through a proactive, risk proportionate and patient-centred approach, with high levels of transparency and engagement of civil society. In the European Union, pharmacovigilance is now fully integrated into the life cycle of medicinal products, with the planning of pharmacovigilance activities commencing before a medicine is placed on the market, and companies
\end{abstract}

Aniello Santoro

aniello.santoro@ema.europa.eu

1 Inspections and Human Medicines Pharmacovigilance and Committes Division, Pharmacovigilance and Epidemiology Department, European Medicines Agency, 30 Churchill Place, London E14 5EU, UK

2 Health Products Regulatory Authority, Dublin, Ireland

3 EMA Pharmacovigilance and Risk Assessment Committee, London, UK

4 Medicines and Healthcare Products Regulatory Agency, London, UK encouraged to start planning very early in development for high-innovation products. After authorisation, information on the safety of medicines continues to be obtained through a variety of sources, including spontaneous reports of adverse drug reactions or monitoring real-world data. Finally, the measurement of the impact of pharmacovigilance activities, auditing and inspections, as well as capacity building ensure that the system undergoes continuous improvement and can always rely on the best methodologies to safeguard public health.

\section{Key Points}

The European Union (EU) pharmacovigilance system ensures the promotion and protection of public health through a proactive, transparent, risk proportionate and patient-centred approach.

The Pharmacovigilance Risk Assessment Committee is at the core of the operations of the EU pharmacovigilance system and is responsible for assessing and monitoring the safety of medicines in the EU.

Enhancing involvement of patients, increasing the EU capacity to use real-world data, developing new scientific methods, achieving better pharmacovigilance for medication errors and the simplification of processes are future drivers of the system. 


\section{Introduction}

Pharmacovigilance [1] is defined as the science and activities relating to the detection, assessment, understanding and prevention of adverse effects or any other drug-related problem. Its objectives are to promote the safe and effective use of drugs by providing reliable information for the assessment of the benefit-risk profile of marketed medicines and the minimisation of risk. Although medicinal products are authorised after their quality, safety and efficacy have been assessed and their benefit-risk balance judged to be positive, the information obtainable before a medicine is placed on the market is ultimately limited and the complete knowledge of the benefit-risk of a medicine can only be further defined after its approval, taking account of evidence gathered in 'real-world' usage.

While medicines bring huge benefit to society by saving lives and reducing suffering, adverse drug reactions (ADRs) represent a major cause of illness and death. In 2008, which is the year that most recent relevant data are available, it was estimated that ADRs accounted for $5 \%$ of hospital admissions and about 197,000 deaths per year in the European Union (EU), thus resulting in a societal cost of $€ 79$ billion [2]. Therefore, activities aimed at reducing this burden can generate a significant positive impact both at the individual and societal level and are critical for the appropriate functioning of any health system.

The EU pharmacovigilance system is underpinned by a regulatory network made up of the national competent authorities (NCAs), the European Medicines Agency (EMA), the European Commission (EC) and a legal framework that establishes roles and responsibilities, principles and procedures [3]. Pharmacovigilance is a key pillar of medicine regulation, in conjunction with support for product development, authorisation, manufacturing oversight, inspection and compliance. The EC is the competent authority for centrally authorised products (CAPs), i.e. medicines that fall under the scope of Regulation (EC) No. 726/2004. After a scientific evaluation carried out by the EMA working through its committees, CAPs receive a marketing authorization valid in all EU Member States (MSs).

The NCAs are the competent authorities responsible for nationally authorised products (NAPs), i.e. medicines that fall under the scope of Directive 2001/83/EC. Nationally authorised products receive from NCAs marketing authorisations valid on their territory, with the EMA involved in the coordination of certain activities, notably pharmacovigilance (Fig. 1).

The EU pharmacovigilance system has at its core the EMA Pharmacovigilance Risk Assessment Committee (PRAC) [4], which includes members appointed by the EU
MSs, independent experts appointed by the EC, as well as representatives of healthcare professionals (HCPs) and patients. While promoting the availability of new medicines to respond to unmet medical needs without delay, the EU pharmacovigilance system has the responsibility to protect patients, by ensuring that the safety of medicines on the market is under continual review (Fig. 2). Nearly all innovative products are authorised through the centralised system, which results in an authorisation valid in all MSs and therefore provides potential access to new medicines for more than 500 million EU citizens.

With the main objective to reduce the health burden of ADRs, the EU pharmacovigilance system underwent a major rationalization and strengthening in July 2012 through the implementation and operationalisation of the revised pharmacovigilance legislation. ${ }^{1}$ The legislation was accompanied by the Commission Implementing Regulation (EU) No. 520/2012, a legally binding act that provides details on operational aspects. Finally, a set of Good Pharmacovigilance Practices (GVP) for the conduct of pharmacovigilance in the EU has been developed by experts from the EMA and NCAs, as guidance to support implementation of and compliance with the legal requirements set in the legislation [5]. The implementation of the above legislation aims at delivering a strengthened EU pharmacovigilance system whose core characteristics are highlighted in Fig. 3.

The last 10 years have seen a paradigm shift in pharmacovigilance from a reactive system responding to emerging risks, to a planned, proactive and risk proportionate approach. Pharmacovigilance is now integrated into the life cycle of a product with the planning of pharmacovigilance activities taking place before the product is authorized for the EU market. The EU pharmacovigilance system delivers a continuum from planning of risk management activities during pre-marketing through to inspections and capacity building (Fig. 4).

On the occasion of the fifth anniversary of the implementation of the revised pharmacovigilance legislation, this review aims at providing a comprehensive overview of the EU pharmacovigilance system and its main components, from the perspective of some of those working at its core. Acknowledging the intrinsic complexity of the EU system, this review could serve as a reference for those new or unfamiliar with it, as well as support future research aimed at analysing the system and its performance. To this end, the article details key aspects of the system.

\footnotetext{
$\overline{1}$ Regulation (EU) No. 1235/2010 and Directive 2010/84/EU, which amended the existing Regulation (EC) 726/2004, and Directive 2001/83/EC, respectively.
} 


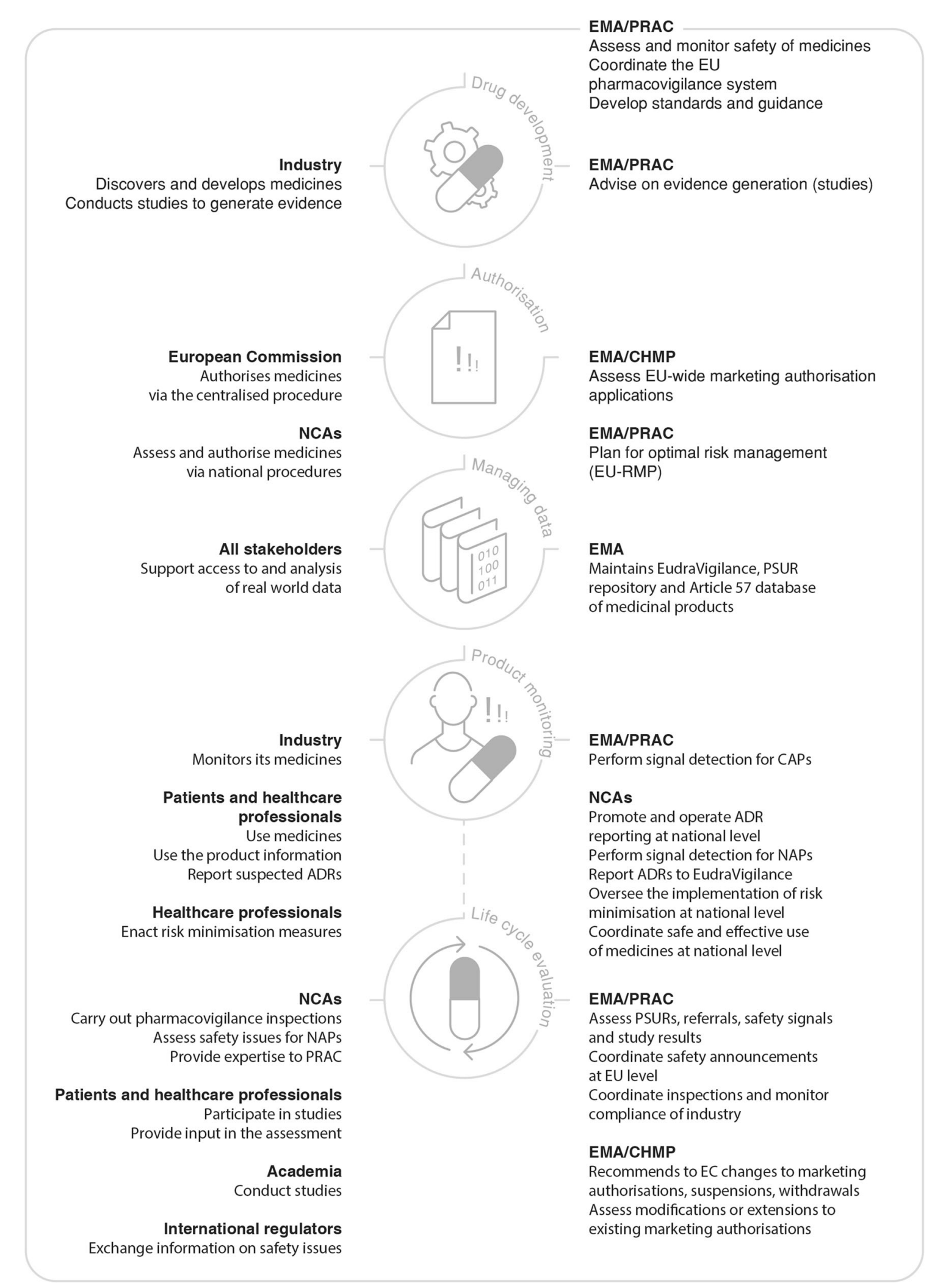

Fig. 1 European Union (EU) pharmacovigilance system: roles and responsibilities through the product life cycle. ADRs adverse drug reactions, CAPs centrally authorised products, CHMP Committee for Medicinal Products for Human Use, EC European Commission, EMA
European Medicines Agency, EU European Union, NAPs national authorised products, NCAs national competent authorities, PRAC Pharmacovigilance Risk Assessment Committee, PSUR periodic safety update report, $R M P$ risk management plan 
Fig. 2 Promotion and protection of public health by the European Union pharmacovigilance system

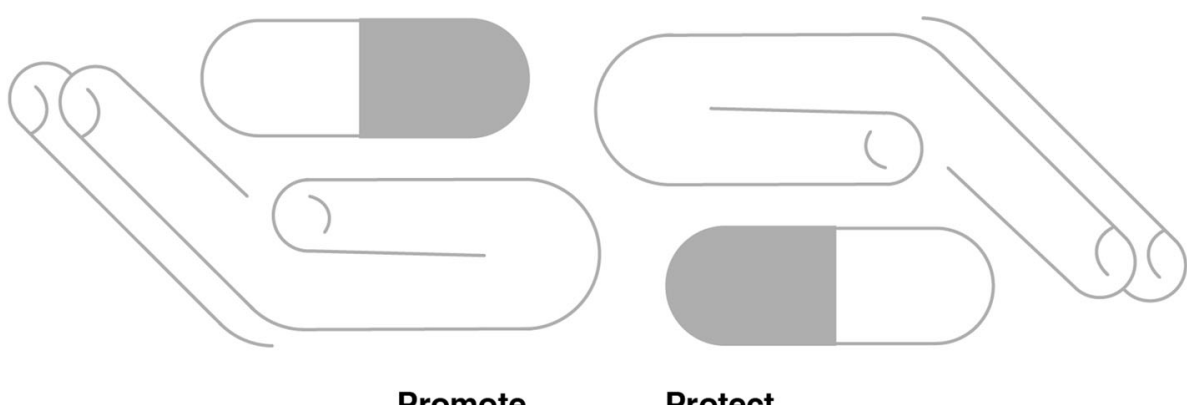

Promote

Fulfill unmet medical needs

Plan evidence generation through lifecycle

Plan for optimal risk management at authorisation

\section{Robust pharmacovigilance \\ systems support \\ authorisation decision}

\section{Robust monitoring for new safety issues}

Rapid decision making

Effective action to minimise risk

Demonstrating positive impact

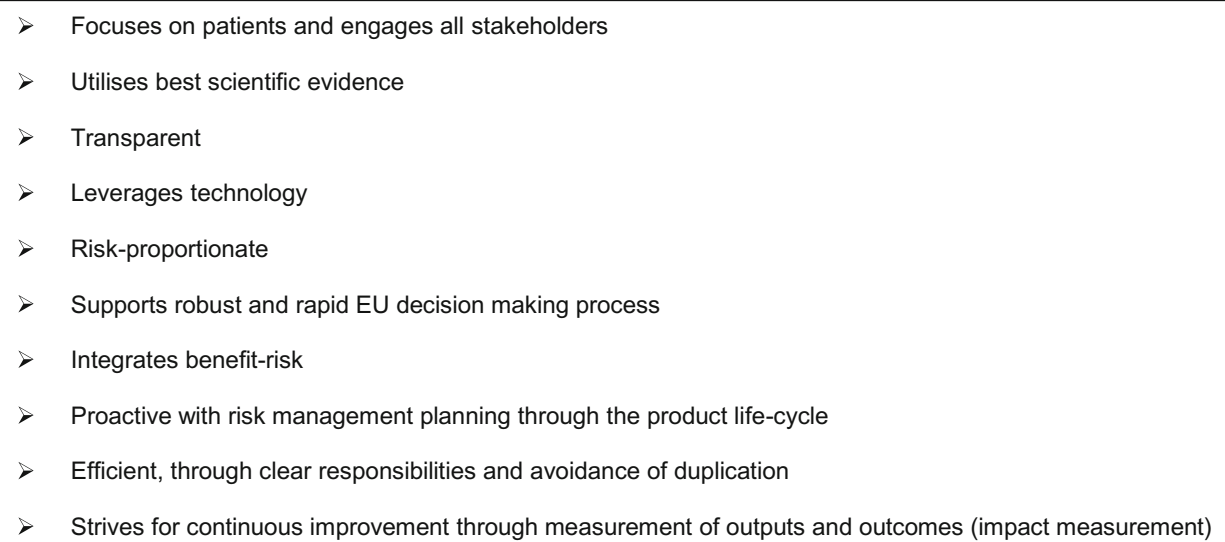

Fig. 3 Core characteristics of the European Union (EU) pharmacovigilance system

Fig. 4 Continuum of the European Union pharmacovigilance system from pre- to post-marketing. $A D R$ adverse drug reaction

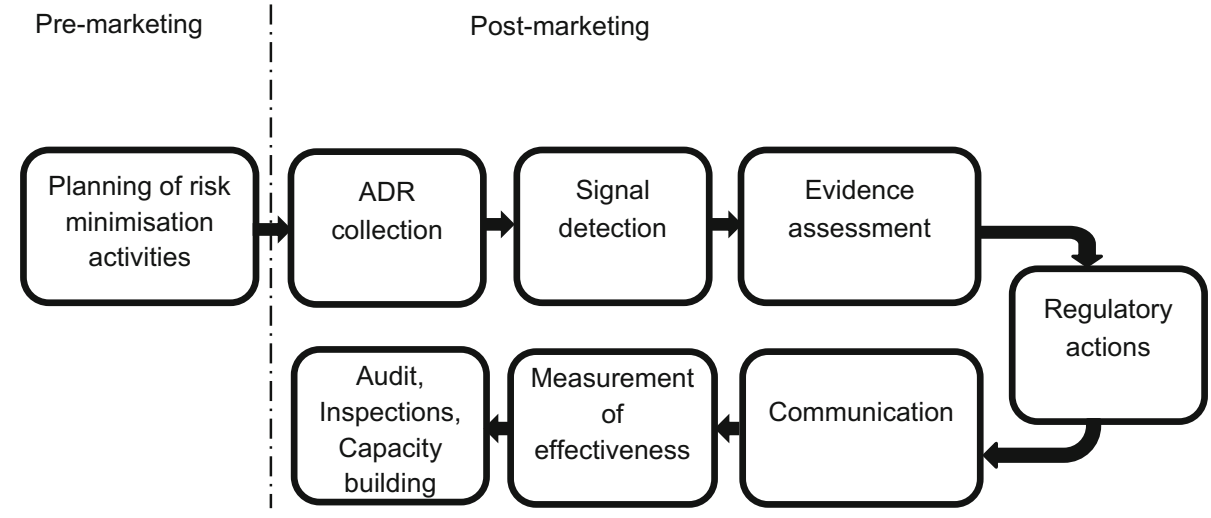




\section{Pharmacovigilance Risk Assessment Committee}

The PRAC is central to the design and operation of the EU system. The PRAC is the committee responsible for assessing all aspects of the risk management of medicines for human use, having due regard to the therapeutic effect of the medicinal product [4]. This includes the detection, assessment, minimisation and communication relating to the risk of adverse reactions, while taking the therapeutic effect of the medicine into account.

It also has responsibility for the design and evaluation of post-authorisation safety studies (PASSs) and pharmacovigilance audits. The decisions by PRAC on imposed non-interventional PASS are directly binding. The PRAC convenes each month to assess risk management plans (RMPs), signals, in-depth reviews of safety issues and benefit-risk of medicinal products, and to prepare recommendations on any questions relating to pharmacovigilance activities and to risk-management systems, including the monitoring of their effectiveness.

Recommendations by PRAC are considered by the Committee for Medicinal Products for Human Use when it adopts opinions for medicines, which include a CAP and by the Coordination Group for Mutual Recognition and Decentralised Procedures-Human when it provides a recommendation on medicines authorised only in the MSs. The past 5 years of experience with PRAC have established the Committee as crucial in taking forward strategic issues in pharmacovigilance, driving improvements in the science of pharmacovigilance and strengthening its processes.

\section{Pharmacovigilance Planning}

Medicinal products are authorised on the basis that their benefit-risk profile is considered positive for the target population and in the specified indication. However, not all risks will have been identified at the time of the initial marketing authorisation and some will only be discovered and characterised in the post-authorisation phase [6].

The planning of pharmacovigilance activities starts long before a medicine is placed on the EU market. Companies are encouraged to plan, from very early in a product's lifecycle, how they will further characterise and minimise the risks associated with the post-authorisation phase. In addition, companies are required by legislation to submit a RMP [7] to regulatory authorities when applying for a marketing authorisation.

The RMP should be updated throughout the lifetime of the medicine, at the request of a regulatory authority, or whenever the risk-management system is modified, for example, as new information that may change the benefit- risk profile of a medicine becomes available, or as a result of an important pharmacovigilance milestone being reached such as completion of a PASS.

The aim of the RMP is to reduce uncertainties regarding the safety profile at different points in the life cycle of a medicinal product and to plan risk management activities accordingly. The RMP provides information on the medicine's safety profile and the risks that need to be further characterised or managed ('safety specification'), as well as on the planning of studies or other pharmacovigilance activities to increase the knowledge on the safety and efficacy of the medicine ('pharmacovigilance plan'). Additionally, in the 'risk minimisation plan', the RMP covers the planning and implementation of risk minimisation measures. This includes the evaluation of the effectiveness of these activities through the life cycle of the product, to ensure that the burden of ADRs is minimised and the overall benefit-risk balance is optimised.

The risk management system needs to be proportionate to the identified and potential risks of the medicine, and the need for post-authorisation data. For instance, the EU pharmacovigilance system includes provisions for the marketing authorisation to include in the risk management plan, e.g. legally binding conditions for PASS, post-authorisation efficacy studies and/or additional risk minimisation activities.

These may take the shape of requesting the marketing authorisation holder (MAH) to provide an educational pack aimed to increase the physicians' awareness on the potential risk of bleeding and to offer guidance as to how to manage that risk, such as in the case of rivaroxaban [8]. In other cases, such as for bosentan, the MAH may be requested to implement with the NCAs a controlled distribution system to ensure that prescribers receive a 'prescriber kit' [9]. This has the objective to raise awareness among prescribers and patients on the teratogenicity, hepatoxicity, contraindications, and effect of the drug on the haemoglobin and fertility, as well as on the need for specific measures and monitoring to mitigate those risks. Additionally, for bosentan, the MAH is also requested to set up a surveillance programme/registry and a PASS to collect specific information on the use of the drug in the context of digital ulcers.

The feasibility of implementing additional risk minimisation activities in all MSs and the challenges posed by different healthcare settings need to be considered and built into the planning. Risk management plans for CAPs are assessed by the PRAC, with possible consultation of HCPs and patients, on a case-by-case basis, especially with regard to proposed risk minimisation measures. Risk management plans for NAPs are assessed by NCAs and PRAC advice may be sought on a case-by-case basis. 
To encourage risk management planning even earlier in the development of innovative medicinal products, in 2015, the EMA expanded the scope of scientific advice [10] to cover also PASS. The advice builds on the expertise of the PRAC and helps improve the design of studies meant to collect further information on the safety of medicines on the market. Scientific advice is elaborated through the EMA Scientific Advice Working Party with PRAC endorsement. This initiative responds to the objective to integrate pharmacovigilance into the planning through the life cycle of products, and therefore supports proactive pharmacovigilance.

As both a transparency measure and to encourage ADR reporting, medicinal products that are being monitored particularly closely by regulatory authorities are included in the list of medicines under additional monitoring [11] that is published on the EMA website and reviewed monthly by the PRAC. Close monitoring is accomplished by the EMA, for instance, by screening EudraVigilance (EV) at increased frequency for ICSRs reported with CAPs included in the list (unless the only reason for inclusion is the requirement for a PASS). The main goal of the list is to promote the collection of additional information as early as possible on products with newly emerging safety concerns or whose safety profile might need further characterisation.

As of April 2017, the list includes over 300 products. New biologicals, medicines containing a new substance, medicines for which a requirement for a PASS is in place, those with specific obligations, those authorised under exceptional circumstances or granted a conditional approval fall in this category. These medicines are identified by an inverted black triangle in their package leaflet and Summary of Product Characteristics. After inclusion in the list, a medicine remains under 'additional monitoring' for 5 years, or until the PRAC decides otherwise (notably when specific studies are completed). In line with a legal requirement, ${ }^{2}$ the EMA is reviewing the use of the list and investigating whether the inclusion of products on it had an effect on ADR reporting.

\section{Collecting Information on Medicines in Clinical Use}

\subsection{EudraVigilance Database of Spontaneous Reports}

Patients and HCPs in the European Economic Area (EEA) can submit reports on suspected ADRs to the relevant NCAs or MAHs. These reports are then transmitted to EV

$\overline{2}$ Article 23(4a) of Regulation (EC) No. 726/2004, as amended.
[12], a centralised database supporting safety monitoring and the safe and effective use of medicines in the EU.

NCAs have a pivotal role in encouraging and facilitating ADR reporting at the national level, while the EU network provides support through the delivery of guidance, tools and the sharing of good practices. EudraVigilance is maintained by the EMA on behalf of the EU network [13].

The EU pharmacovigilance system supports high levels of transparency in pharmacovigilance in general and for ADR reporting specifically. Therefore, the general public has access to anonymised data from EV through the website www.adrreports.eu. Patients and HCPs can now access information in all official EU languages including the number of serious cases submitted spontaneously to EV for CAPs, as well as for common substances contained in nationally authorised medicines [14].

EudraVigilance will be further enhanced with the release of new functionalities on 22 November, 2017. These functionalities will include delivering improved quality and completeness of individual case safety reports (ICSRs) based on the new International Organization for Standardization standard for ICSRs [15]. The new system will also have the added capability of dealing with an expected higher number of reports, as a consequence of the new requirement to report non-serious cases and of the increase in reports of suspected ADRs from patients. Moreover, it will enhance signal detection capabilities by granting EV access to MAHs (according to the revised EV access policy [16]), and it will reduce duplications of efforts by establishing simplified reporting by MAHs to EV only with re-routing of ICSRs to MSs, and to the World Health Organization Uppsala Monitoring Centre.

Scientific and medical literature is another important source of information to identify suspected ADRs. The EU pharmacovigilance system requires the EMA to establish and maintain a literature monitoring service. This has been fully operational since September 2015. This service currently monitors selected medical literature for a subset of commonly used chemical and herbal substance groups, to identify suspected ADRs and enter the relevant information in EV [17]. These substances have been selected on the basis that a high number of marketing authorisations have been granted to various MAHs in the EEA. These ICSRs are then made available to the NCAs and to the MAHs for download and potential reporting to other international regulators. The literature monitoring service improves data quality and has the potential to enhance the efficiency of ADR reporting, as MAHs do not have to report to EV suspected ADRs recorded in the medical literature monitored by the EMA for products containing the substances included in the list. The service limits duplicative efforts by multiple MAHs, each of whom previously had to monitor the same literature and enter the same case report into 
pharmacovigilance systems and, as a consequence, it reduces the number of duplicate case reports in EV.

The EU pharmacovigilance system and its processes are underpinned by a comprehensive inventory of structured data for all medicines authorised in the EU. Marketing authorisation holders are required to submit information on their authorised medicines and keep it up to date [18] through the 'Article 57' or the 'eXtended EudraVigilance Medicinal Product' database. This supports data analysis and signal management (e.g. identification of products concerned by a signal and of relevant MAHs), regulatory activities [e.g. the coordination of actions to safeguard public health, including referral procedures, establishment of the periodic safety update report (PSUR) repository and literature monitoring] and communication (e.g. between PRAC and MAHs). Over time, the data held will be upgraded to fully comply with the International Organization for Standardization standards on the identification of medicinal products [19-23]. It is expected that this will simplify the exchange of information between all stakeholders, by enhancing interoperability of systems at the EU level and internationally.

\subsection{Real-world Data}

Real-world data refer to data collected in routine clinical practice. For pharmacovigilance, this is particularly significant as they are collected outside the constraints of conventional randomised clinical trials, where most of the data supporting a marketing authorisation come from. There are multiple potential sources of data including electronic health records generated in both the primary and secondary care environments, administrative claims records, prescription databases and registries. Clinical information from these other sources may be used to complement data already available and to more effectively monitor the safety and efficacy of authorised medicines once marketed. However, each of these data sources has strengths and limitations associated with its use and a clear understanding of these is essential to define where and when each data source can add the most value.

It could be argued that amongst these data sources, electronic health record and registries offer the most promise in the context of the regulatory decision making because of the richness of the clinical data they hold. Electronic health records have been used for some time to support decision making by the EU pharmacovigilance system as many are able to provide a detailed longitudinal patient history record, are relatively low cost and linkage with other datasets may be possible if the record includes a unique patient identifier. However, often hospital data are not recorded specifically for such purposes and hence gaining the full picture in specific diseases, especially rare diseases or where care is predominantly delivered in the hospital setting can be challenging. Registries may fill this gap in information emphasising the complementary nature of many of these data sources. Registries are organised systems that collect uniform information over time on patients with a disease, condition or on a particular treatment(s).

However, MAHs and regulators may face challenges in using existing registries, including differences between registries in the types, structure and quality of data provided and data accessibility. To rationalise and streamline the use of existing sources of information during the evaluation of the safety of medicinal products, the EMA has launched in 2016 an initiative on patient registries [24]. This initiative aims at making better use of existing registries as a source of high-quality post-authorisation data for regulatory decision making or, in case a suitable one does not exist, at facilitating the establishment of new registries.

\section{Analysis of data}

\subsection{Signal Management}

European Union regulators have the responsibility to monitor EV data to determine whether there are new risks, or risks have changed and whether those risks impact on the benefit-risk balance of medicinal products authorised in the EU. This is accomplished through the signal management process, which involves signal detection, validation, confirmation, analysis and prioritisation, assessment and, finally, recommendations [25] for regulatory action, including new product labelling, issued by the PRAC.

Signal detection is key within the EU pharmacovigilance system to promote public health by optimising the safety and efficacy of medicines and informing their use. Within the EU pharmacovigilance system, a signal is defined as information that arises from one or multiple sources (including observations and experiments), which suggests a new potentially causal association, or a new aspect of a known association, between an intervention and an event or set of related events, either adverse or beneficial, that is judged to be of sufficient likelihood to justify verificatory action' [26]. The EMA in collaboration with the PRAC rapporteurs leads on signal detection for substances contained in CAPs, while the NCAs lead for substances contained only in NAPs.

Signals may originate from several sources such as spontaneous reports, clinical trials, epidemiological studies [27] and the published literature. EudraVigilance contains information on suspected ADRs reported in association with medicinal products authorised in the EEA, and 
therefore is a key source of signals for regulatory authorities. Reports from HCPs and patients can be retrieved from $\mathrm{EV}$ and analysed as they are received, thus providing valuable information on the real-world use of medicines. Once sufficient evidence on the existence of a new potential causal association (or a new aspect of a known association) is gathered, a signal is validated. The clinical significance of the signal, previous awareness of it, the biological and temporal plausibility and any relevant sources of information supporting the association are taken into consideration during the validation process.

Marketing authorisation holders also perform signal detection for their medicinal products using any data sources available to them (e.g. corporate ADR database, scientific literature), including EV data from November 2017. Currently, MAHs are required to notify the competent authorities in MSs where the product is authorised and to the EMA validated signals that may qualify as emerging safety issues [28], and to submit safety variations, in case an update of the product information is warranted. Validated signals are also presented in the relevant sections of the PSUR [29].

Signal detection methods in the EU have been enhanced based on the results from the IMI PROTECT (Pharmacoepidemiological Research on Outcomes of Therapeutics by a European Consortium) project [30]. New guidance regarding the MAH's signal detection activities is being developed and this focuses on MAHs' access to EV and the consequences for signal detection and management. This is provided in the draft revision 1 of GVP Module IX [28].

Validated signals that have been confirmed by a regulatory authority, undergo a thorough assessment by the PRAC, which involves a rigorous scientific evaluation of all the evidence available, including possibly additional data requested of the MAH(s). This results in a PRAC recommendation that, based on the outcome of the evaluation, may typically range among a series of options (see Fig. 5).

\begin{tabular}{|ll|}
$>$ & Regulatory actions (update of PI, RMP, referral, USR) \\
$>$ & Additional communication (DHPC) \\
$>$ & Additional data (PSUR, PASS) \\
& Ongoing routine pharmacovigilance \\
\hline
\end{tabular}

Fig. 5 Types of Pharmacovigilance Risk Assessment Committee recommendations for signals. DHPC direct healthcare professional communication, PASS post-authorisation safety study, $P I$ product information, $P S U R$ periodic update safety report, $R M P$ risk management plan, USR urgent safety restriction
During 2016, 30\% of the 94 signals prioritised and assessed by PRAC led to a recommendation for a product information update [three of which also included a direct healthcare professional communication (DHPC)], four were escalated into a referral procedure, one required further assessment through a PASS and one led to the update of the RMP, while for 30 signals ongoing routine safety monitoring was considered sufficient [13].

Irrespective of the type of PRAC recommendation, the thorough assessment at a specific point in time of all available information within the framework of a clearly defined signal procedure provides patients with the assurance that all the relevant aspects concerning the ADR of interest have been explored and that the appropriate action is taken. This can be to refute a signal, or to escalate the review to a different formal regulatory procedure, e.g. a referral (in case the full benefit-risk assessment is necessary) or a variation (if a labelling change is warranted) [31], or a combination thereof.

For instance, a signal of potential increased risk of toe amputation in patients taking canagliflozin was triggered by data from two clinical trials (CANVAS and CANVAS$\mathrm{R})$. The PRAC recommended an initial distribution of a DHPC to inform HCPs about these concerns. Meanwhile, a thorough evaluation of the entire class of sodium-glucose co-transporter 2 inhibitors was started via a referral procedure, at the end of which it was concluded that a labelling change to warn patients of this risk and of its possible signs was warranted [32].

In other cases, such as for thiocolchicoside, the assessment of a signal of potential genotoxicity led to a restriction in its use. As a result, medicines containing thiocolchicoside are now only recommended at low doses and for short-term use, as an addition to other treatment [33].

The PRAC's recommendations are published on the EMA website within a month of the respective plenary meeting. Where a product information update is involved, translations of the relevant text in all EU official languages, as well as Norwegian and Icelandic, are also published for direct use by MAHs of innovator and generic products [34]. This service reduces duplication of effort by MAHs by centralising the provision of high-quality translations. It facilitates a speedier update of the product information update in a consistent way across different countries, thereby helping to ensure that clear information is provided to EU patients about their medicines.

\subsection{Periodic Safety Update Reports}

The EU pharmacovigilance system places a legal requirement on MAHs to provide an evaluation of the benefit-risk balance of their medicines at intervals as evidence accrues 
in clinical use. The main way that this is accomplished is by submitting to EU regulatory authorities PSURs at defined time points following a medicine's authorisation [29] throughout its life cycle. A PSUR is a comprehensive, concise and critical analysis of the benefit-risk balance of a medicinal product in clinical practice, including its use in unauthorised indications as well as in line with the product information. Periodic safety update reports take into account new or emerging information in the context of cumulative information on risks and benefits and include the results of all studies carried out with a medicine, in its authorised and unauthorised indications.

The EU pharmacovigilance system requires that the obligations imposed in respect of PSURs are proportionate to the risks posed by medicinal products; therefore, the PSUR reporting cycle is linked to the risk management system of a medicinal product. For instance, following a risk-based approach, the frequency of submission of PSURs may vary from 6 months to several years for different substances. Moreover, the routine submission of PSURs for generic well-established use, homeopathic and traditional herbal medicinal products is waived, unless otherwise specified in the marketing authorisation, or required through the EU reference date list [35] or by a NCA.

While assessing PSURs, regulatory authorities use the information in them to determine whether there are new risks with a medicine or the balance of its benefits and risks has changed, and, if needed, take regulatory actions to protect the public from the risk identified, e.g. via an update of the product information. The outputs of the assessment of PSURs are legally binding on the MAHs.

To increase the efficiency in the use of resources between competent authorities in MSs, the assessment of PSURs containing the same active substance or combination of active substances has been synchronised, through the establishment of the EURD list. This provides harmonised information on data lock points and frequency of submission of PSURs. For substances included in the list, the PRAC performs a single joint assessment of all related PSURs (PSUSA), regardless of the authorisation procedure and the EU country of authorisation of individual products. This translates into a strengthened benefit-risk review of medicines across the EEA, and hence into enhanced safety monitoring and rapid labelling changes across the EU. The EURD list also provides an opportunity for international harmonisation of PSUR submission and, potentially, international collaboration on assessment.

Additionally, to simplify the assessment process, the EMA has established an EU PSUR 'repository' [36]. As of June 2016, MAHs are required to upload PSURs to this repository only (i.e. there is no longer a need for direct submission of individual reports to the individual NCAs).
This is regardless of the product's authorisation procedure and represents a significant simplification of PSUR submissions for industry. The repository is the single central secured platform for PSURs, PSUR assessment reports, comments and final outcomes, thus, ensuring that NCAs, the EMA, its committees and the EC have timely and secure access to all relevant documents to monitor the safety of products authorised in the EU.

\subsection{Formal Review of Important Safety Issues via Referrals}

For the most important pharmacovigilance reviews, be they new safety issues, reviews of the effectiveness of risk minimisation or full benefit-risk reassessments driven by pharmacovigilance concerns, the EU pharmacovigilance system empowers the MSs and the EC to trigger a 'referral procedure'. This means that a medicine or a class of medicines is 'referred' to the EMA and the PRAC is requested to conduct a scientific assessment and make a recommendation on the safety or benefit-risk issue at stake on behalf of the EU [37], in the light of all the evidence available. Relevant information from the literature, including pharmacoepidemiological studies and metaanalyses, from non-clinical studies, spontaneous reports and clinical trials, and any available academic research are fed into the assessment. Referrals offer opportunities for involving scientific advisory groups and for engaging patients and researchers, who can each contribute to the assessment from their specific angle.

A referral can be triggered for products belonging to any therapeutic class, including both established and innovative medicines. Results of referral procedures contribute towards an optimised use of medicinal products. For instance, they have recently led to new recommendations to minimise the risk of diabetic ketoacidosis in patients taking sodium-glucose co-transporter 2 inhibitors, the risk of progressive multifocal leukoencephalopathy in patients taking Tysabri ${ }^{\circledR}$ (natalizumab) or the risk of serious infections in patients treated with Zydelig ${ }^{\circledR}$ (idelalisib).

Information on the commencement of the referral, the timetable, list of affected products and questions for the MAHs is promptly published on the EMA's website [38], thus allowing real-time transparency. Relevant MAHs are notified and asked to contribute to the referral, including the provision of data and information for the PRAC's assessment.

The PRAC recommendation on the safety issue is then forwarded to the Committee for Medicinal Products for Human Use for opinion or, if no CAPs is involved, to the Coordination Group for Mutual Recognition and Decentralised Procedures-Human for its agreement. Once the evaluation is concluded, the PRAC assessment report 
outlining the outcome of the referral procedure is also published on the EMA website.

Safety referrals are a very effective pharmacovigilance instrument to coordinate a thorough and timely review of the safety of products including those authorised via different procedures. Referrals result in a harmonised position across the EU, ratified by a decision that is immediately implementable and legally binding for all the MSs.

\section{Taking Regulatory Action}

Within the scope of its mandate as a statutory committee, the PRAC may recommend different regulatory actions, as a result of the evaluation of a safety issue and based on its urgency, seriousness and public health impact. These have the objective to prevent or minimise harm from ADRs and enable the safe and effective use of medicinal products. These risk minimisation measures aim to provide information for patients and practitioners, or to restrict the use of the medicine.

For instance, the PRAC may recommend an update of the product information (e.g. to include a particular ADR, to require to perform a patient monitoring test or to introduce a new warning or contraindication), with the objective to increase awareness of specific ADRs. Alternatively, the PRAC may recommend other routine risk minimisation measures (e.g. controlling the number of dosage units, modifying the legal status of the medicine, or restricting its administration to hospitals or particular HCPs), when it is deemed that restricting access to the medicine will minimise the occurrence of an ADR. Additionally, the PRAC may recommend specific communication measures, for example, a DHPC or other additional risk minimisation activities [39] such as the setting up of a $\mathrm{HCP} /$ patients' educational programme or of a pregnancy prevention programme, when for instance a product displays a potential teratogenic effect and it is essential to minimise the occurrence of pregnancy during exposure. In the most serious cases, when the benefit-risk balance for a medicine is considered to have become negative and warnings and restrictions are judged not to be sufficient, the PRAC may recommend the suspension or revocation of the marketing authorisation, leading to product withdrawal from the market.

\section{Transparency, Participation, Communication and International Collaboration}

Recent years have seen an unprecedented increase in the level of transparency of the EU pharmacovigilance system. Through the EMA website, the public now has access to very extensive information on EU pharmacovigilance (Fig. 6).

Summaries of RMPs for any newly authorised CAP are published together with the other documents of the European Public Assessment Report for that medicine. Whenever the RMP of a medicine includes additional risk minimisation measures to prevent medication errors, a communication in all EU official languages forms part of the European Public Assessment Report, as well as being published on a dedicated EMA webpage [40], to raise awareness among HCPs and patients.

Similarly, the protocols and abstracts of the final study reports of imposed PASSs are published in the EU postauthorisation study register [41]. The EU pharmacovigilance system is underpinned by an increased participation of civil society in the proceedings of scientific committees, with representatives of patients, as well as of HCPs being members of the PRAC, the Pediatric Committee, the Committee on Orphan Medicinal Products and the Committee for Advanced Therapies. This enriches the scientific discussion with valuable input from the user community and the clinical world. Additionally, with the promotion of patient reporting of suspected ADRs and the publication of the list of medicines under additional monitoring, patients are more empowered because they are given further opportunity to be engaged with and to contribute to the safety of medicines.

The EU pharmacovigilance system provides the PRAC with the possibility to hold public hearings as a tool to engage EU citizens in the process of decision making on key safety issues and to listen to their views and experiences [42]. As of April 2017, the PRAC has not held any public hearing; however, they may take place when the PRAC establishes that collecting the views of the public would bring added value to its review. Public hearings will be open to all members of the public, while MAHs also have the opportunity to present their views. Contributions made by the public will be considered by the PRAC and inform the Committee's decision-making process, especially with regard to the acceptability of the risks of a medicine in relation to its therapeutic effects and the available therapeutic alternatives, and the feasibility and acceptability of risk minimisation activities. They will be announced on the EMA's website, with a list of questions and information on how to participate.

In parallel, the EMA has also strived to strengthen its communication channels. For instance, MAHs receive advance notification of safety signals to alert them to providing prompt and comprehensive support to the assessment process. Concerned MAHs also receive the preliminary assessment report on the data that they have submitted in the context of the PRAC assessment of signals and, where a product information update is proposed, they 
Fig. 6 Some of the European Union pharmacovigilance information published on the European Medicines Agency (EMA) website. CAPs centrally authorised products, EPAR European Public Assessment Report, EV EudraVigilance, $N A P s$ nationally authorised products, PRAC Pharmacovigilance Risk Assessment Committee

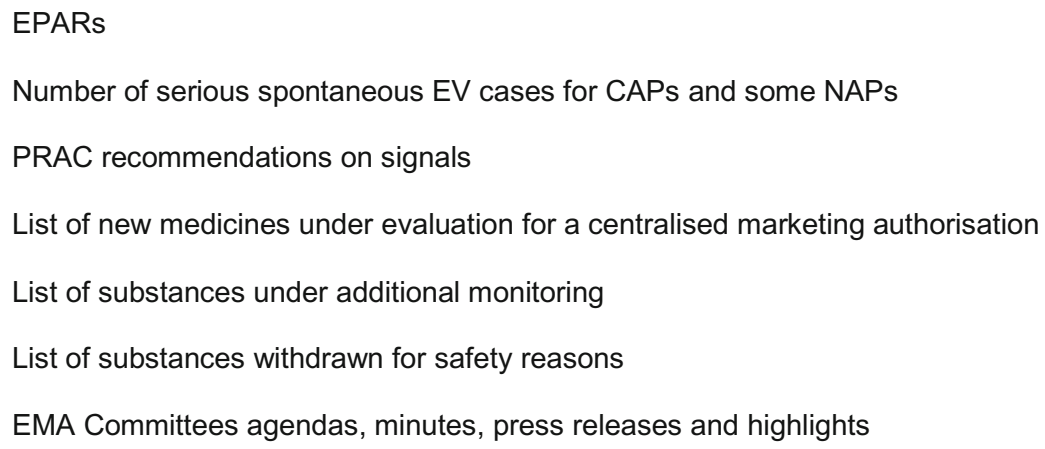

are given the opportunity to comment and contribute to the final wording. In the case of referrals, when a DHPC is recommended, or if major regulatory actions (e.g. suspension, revocation of the marketing authorisation, restriction of indication) are taken as a result of the assessment of a safety issue, in addition to usual communication tools with e.g. MAHs and HCPs, the EMA also publishes a press release to ensure a wider outreach of the message.

For active substances contained in medicinal products authorised in more than one MS, the EMA is responsible for coordinating safety announcements between NCAs. This ensures that a harmonised and consistent message is provided at the same time to patients and HCPs across the EU. When a safety issue is likely to attract media interest, the EMA may additionally prepare 'lines-to-take', i.e. standardised briefing text as a resource for the EU network, when responding to enquires following the dissemination of a safety announcement [43].

Over the past few years, the EMA has significantly increased its level of collaboration and information sharing with international regulators to advance regulatory excellence worldwide [44]. Since 2013, information on risk assessments, anticipated regulatory actions and inspection findings, as well as views on the public health impact, priorities and goals of pharmacovigilance activities are regularly shared via teleconferences between the US Food and Drug Administration and the EMA, with Japan and Canada as observers. This supports regional risk assessment, enriches the decision-making phase and facilitates international coordination of regulatory action, including the timing of public communication [45].

Additionally, to further increase cooperation among pharmacovigilance stakeholders and promote efficiency, a confidentiality arrangement has been established between the EMA, the EC and the World Health Organization [46] since 1 September, 2015. Under its terms, post-authorisation pharmacovigilance data, as well as safety concerns arising from PSURs, post-authorisation obligations and commitments, information in applications for scientific advice, post-authorisation activities of significant public health interest and data related to inspections may be shared between these organisations. To achieve a truly global perspective, the EMA, the EC and the World Health Organization can integrate information they may have in house with that available elsewhere. This enables earlier detection of new or changing safety issues and more timely regulatory action, it avoids duplication of assessments and improves the authorisation and safety of medicines, by allowing pooling of information and expertise, the overarching goal being patients' safety and their accelerated access to new and innovative medicines.

\section{Quality Management System}

To fulfill the requirements of the legislation, MAHs, NCAs and the EMA have to establish and use adequate and effective quality systems. A quality system encompasses the setting up of structures and processes (quality planning), the carrying out of tasks and responsibilities in accordance with quality requirements (quality adherence), the monitoring and evaluation of effectiveness of structures and processes (quality control and assurance) and the correction and improvement when necessary (quality improvements) [47]. The overall quality objectives of a pharmacovigilance system are to (1) comply with the task and responsibilities set up in the legislation, (2) prevent ADRs and (3) promote the safe and effective use of medicinal products, thus contributing to public health.

For all authorised medicinal products, MAHs are required to maintain a description of their pharmacovigilance system in the pharmacovigilance system master file, which includes information relating to the persons (e.g. qualified person responsible for pharmacovigilance in the EU), as well as of the procedures put in place to ensure the safety of medicines. A copy of the pharmacovigilance 
system master file can be requested at any time and is usually requested during pharmacovigilance inspections.

The EMA and the MSs need to cooperate and maximise their resources to continuously develop pharmacovigilance systems capable of achieving high standards of public health protection for all medicinal products, regardless of the routes of marketing authorisation. Additionally, to facilitate interaction between NCAs, the EMA, the EC and MAHs, competent authorities have to keep accessible descriptions of their organisational structures, assignment of tasks and responsibilities, and contact points.

\section{Monitoring of the European Union Pharmacovigilance System}

The monitoring of the EU pharmacovigilance system is key to driving overall process improvements, and to enhancing the risk minimisation of specific products. Measuring the impact of pharmacovigilance activities involves measuring the effectiveness of specific risk minimisation measures, as well as monitoring the compliance of MAHs with their pharmacovigilance obligations and the auditing and inspection of pharmacovigilance systems.

The evaluation of risk minimisation measures is an essential aspect of continuous pharmacovigilance, both in terms of their actual implementation and of their effectiveness in achieving the desired outcome, e.g. a reduction in the burden of an ADR, to demonstrate public health protection [48]. Different methodologies may be used to perform these evaluations. For instance, the PRAC may request MAHs to initiate a PASS to obtain further information on the safety of a medicine, to identify, characterise or quantify a safety hazard but also to measure the effectiveness of a risk minimisation measure. By measuring the impact of pharmacovigilance activities, regulators can determine what activities are successful and identify enablers and barriers for generating positive impacts, which contribute to the development of an EU proactive pharmacovigilance system [49].

To achieve this objective, the PRAC adopted in 2016 a strategy [49] that builds on existing activities in MSs and EMA and relies on a collaborative approach with stakeholders. This strategy focuses on four areas (Fig. 7) and is complemented by specific activities and deliverables for the subsequent 3 years [50].

The auditing and inspections of pharmacovigilance systems are conducted both routinely and when there is a suspicion that the requirements for a pharmacovigilance system are not met [51]. National competent authorities have the legal responsibility of supervisory authorities to ensure compliance of MAHs with pharmacovigilance obligations and they carry out inspections of their

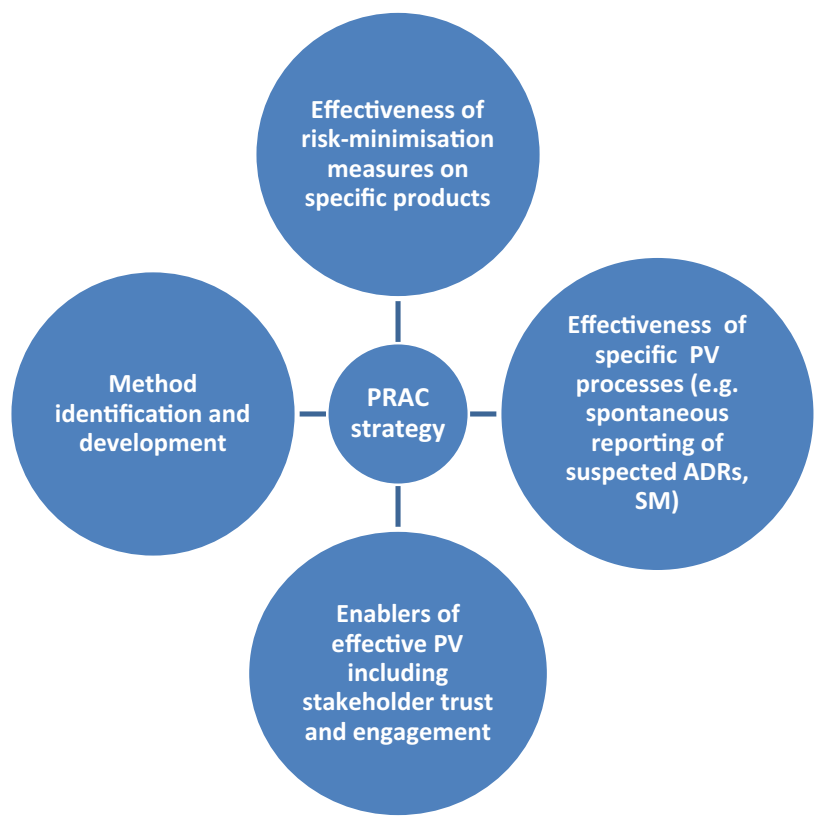

Fig. 7 Areas of the Pharmacovigilance Risk Assessment Committee (PRAC) strategy to measure the impact of pharmacovigilance activities. $A D R$ adverse drug reaction, $P V$ pharmacovigilance, $S M$ signal management

pharmacovigilance systems to this effect. The EMA plays a coordinating and facilitating role by providing support for harmonisation and guidance at the EU level. This ensures that a consistent approach is followed throughout the EU and facilitates the subsequent undertaking of concerted actions.

\section{Capacity Building}

An important area is capacity building, as a way to ensure that the EU pharmacovigilance system operates optimally, undergoes continuous improvement and can always rely on the best methodologies to safeguard public health. In addition to measuring the impact of pharmacovigilance activities, capacity building takes the form of the development of evidence-based methodologies, e.g. in the context of the IMI PROTECT project [52], which delivered on improving early and proactive signal detection from various data sources, enhancing data collection from consumers, developing the methodological framework for pharmacoepidemiological studies, and enabling the integration and presentation of data on benefits and risks.

Another aspect of capacity building is represented by offering continuous training to pharmacovigilance partners and stakeholders. This is achieved by making information on meetings, events and public consultations promptly available on the EMA website [53], as well as through the activity of the EU Network Training Centre [54] whose 
mission is to ensure that good scientific and regulatory practices are spread across the EU network along with harmonised training standards.

Likewise, the European Commission Joint Action Strengthening Collaboration for Operating Pharmacovigilance in Europe (SCOPE) [55] project was set up in 2014 to promote best practice approaches in the EU network by developing guidance and training in key aspects of pharmacovigilance, along with tools and templates to support good practice and an exchange programme for assessors. Additionally, capacity building is achieved by ensuring access to data, information and knowledge, and supporting networking including networks of excellence such as ENCePP [56] aimed at facilitating the conduct of multicentre independent studies focusing on safety and on the balance of benefits and risks of medicines.

\section{Future Priorities}

In the future, the EU pharmacovigilance system will focus on enhancing further the involvement of patients and increasing the EU capacity for real-world evidence by harnessing the power of technology in using tools offered by health informatics (eHealth) or mobile devices (mHealth), e.g. to collect aggregate and patient-level health data. The measurement of the effectiveness of risk minimisation measures will become more routinely embedded into pharmacovigilance practices. This, coupled with the development and deployment of new scientific methods and further simplification in processes, will provide the EU system with more streamlined approaches to monitor the safety of medicinal products and address specific areas of interest such as medication errors, off-label use or use in special populations.

\section{Conclusions}

Since it was first introduced, pharmacovigilance has continuously evolved. Initially based mainly on the analysis of case reports, effective in detecting serious and rare ADRs [57], pharmacovigilance has evolved into a quality systems-based scientific discipline and set of activities that include the whole spectrum of evidence relevant to monitoring medicines on the market. The EU pharmacovigilance system embraces this broad scope and is founded on a proactive, risk proportionate and above all patient-centered approach to promoting and protecting public health.

This public health-oriented system is characterized by responsiveness to new and emerging safety information, and continual effectiveness monitoring in an environment of transparency and openness. The system also strives for increased efficiency, by focussing on what works, streamlining processes and reducing duplication, with more efficiency efforts to be seen in the coming years.

The evolution of the EU pharmacovigilance system during the last 10 years and especially after 2012 has had the achievement of better and faster decisions for medicine use as its main goal. Pivotal to this is the provision of upto-date information and warnings to patients. As stated in the introduction, it is acknowledged that the EU pharmacovigilance system may appear complex and the current article may be seen as an effort by those within the system to inform the public of the key aspects that work together to achieve the goal of safe and effective use of medicines to the benefit of public health.

Acknowledgements The authors gratefully acknowledge Radoslav Zhelev for his contribution to the graphics in the article and Kevin Blake for the critical review of the draft.

\section{Compliance with Ethical Standards}

Funding No sources of funding were used to assist in the preparation of this review.

Conflict of interest Aniello Santoro, Georgy Genov and Peter Arlett are employees of the European Medicines Agency. Almath Spooner and June M. Raine are members of the Pharmacovigilance Risk Assessment Committee, as well as employees of the Health Products Regulatory Authority and the Medicines and Healthcare Products Regulatory Agency, respectively.

Disclaimer The views expressed in this article are the personal views of the authors and may not be understood or quoted as being made on behalf of or reflecting the position of the agencies or organizations with which the authors are affiliated.

Open Access This article is distributed under the terms of the Creative Commons Attribution-NonCommercial 4.0 International License (http://creativecommons.org/licenses/by-nc/4.0/), which permits any noncommercial use, distribution, and reproduction in any medium, provided you give appropriate credit to the original author(s) and the source, provide a link to the Creative Commons license, and indicate if changes were made.

\section{References}

1. World Health Organization. Pharmacovigilance. http://www. who.int/medicines/areas/quality_safety/safety_efficacy/pharmvigi/ en/ (2017). Accessed 3 Jul 2017.

2. European Commission. MEMO/08/782, Brussels, 10 December November 2008. Strengthening pharmacovigilance to reduce adverse effects of medicines. http://europa.eu/rapid/press-release_ MEMO-08-782_en.htm?locale=en (2017). Accessed 3 Jul 2017.

3. European Commission. Pharmacovigilance. http://ec.europa.eu/ health/human-use/pharmacovigilance/index_en.htm

(2017). Accessed 3 Jul 2017.

4. European Medicines Agency. Pharmacovigilance Risk Assessment Committee (PRAC). http://www.ema.europa.eu/ema/index. jsp?curl=pages/about_us/general/general_content_000537.jsp (2017). Accessed 3 Jul 2017. 
5. European Medicines Agency. Good pharmacovigilance practices. http://www.ema.europa.eu/ema/index.jsp?curl=pages/regulation/ document_listing/document_listing_000345.jsp (2017). Accessed 3 Jul 2017.

6. European Medicines Agency. Guideline on good pharmacovigilance practices (GVP): module V: risk management systems (Rev 2). http://www.ema.europa.eu/docs/en_GB/document_library/ Scientific_guideline/2012/06/WC500129134.pdf (2017). Accessed 3 Jul 2017.

7. European Medicines Agency. Risk-management plans. http:// www.ema.europa.eu/ema/index.jsp?curl=pages/regulation/docu ment_listing/document_listing_000360.jsp (2017). Accessed 3 Jul 2017.

8. European Medicines Agency. Xarelto: rivaroxaban. Available from: http://www.ema.europa.eu/ema/index.jsp?curl=pages/medi cines/human/medicines/000944/human_med_001155.jsp\&mid= WC0b01ac058001d124 (2017). Accessed 3 Jul 2017.

9. European Medicines Agency. Tracleer: bosentan. http://www. ema.europa.eu/ema/index.jsp?curl=pages $/$ medicines $/$ human $/$ medi cines/000401/human_med_001100.jsp\&mid=WC0b01ac058001 d124 (2017). Accessed 3 Jul 2017.

10. European Medicines Agency. Post-authorisation safety studies: questions and answers. http://www.ema.europa.eu/ema/index. jsp?curl=pages/regulation/q_and_a/q_and_a_detail_000134.jsp\& mid=WC0b01ac0580796d88 (2017). Accessed 3 Jul 2017.

11. European Medicines Agency. List of medicines under additional monitoring. Available from: http://www.ema.europa.eu/ema/ index.jsp?curl=pages/regulation/document_listing/document_ listing_000366.jsp\&mid=WC0b01ac058067c852 (2017). Accessed 3 Jul 2017.

12. European Medicines Agency. EudraVigilance. http://www.ema. europa.eu/ema/index.jsp?curl=pages/regulation/general/general_ content_000679.jsp\&mid=WC0b01ac05800250b5 (2017). Accessed 3 Jul 2017.

13. European Medicines Agency. 2016 annual report on EudraVigilance for the European Parliament, the Council and the Commission. Available from: http://www.ema.europa.eu/docs/en_GB/ document_library/Report/2017/03/WC500224056.pdf (2017). Accessed 3 Jul 2017.

14. European Medicines Agency. European database of suspected adverse drug reaction reports. http://www.adrreports.eu/en/ (2016). Accessed 3 Jul 2017.

15. International Organization for Standardization. ISO/HL7 27953-2:2011, health informatics: individual case safety reports (ICSRs) in pharmacovigilance. https://www.iso.org/standard/ 53825.html (2016). Accessed 3 Jul 2017.

16. European Medicines Agency. European Medicines Agency policy on access to EudraVigilance data for medicinal products for human use (EudraVigilance access policy), revision 3. http:// www.ema.europa.eu/docs/en_GB/document_library/Other/2016/ 12/WC500218300.pdf (2016). Accessed 3 Jul 2017.

17. European Medicines Agency. Monitoring of medical literature and entry of adverse reaction reports into EudraVigilance. http:// www.ema.europa.eu/ema/index.jsp?curl=pages/regulation/general/ general_content_000633.jsp\&mid=WC0b01ac05808ce84c (2017). Accessed 3 Jul 2017.

18. European Medicines Agency. Data submission on medicines (article 57). Available from: http://www.ema.europa.eu/ema/ index.jsp?curl=pages $\% 2$ Fregulation $\% 2$ Fgeneral\%2Fgeneral_ content_000496.jsp (2017). Accessed 3 Jul 2017.

19. International Organization for Standardization. ISO 11238:2012, health informatics: identification of medicinal products. Data elements and structures for the unique identification and exchange of regulated information on substances. https://www. iso.org/standard/55031.html (2014). Accessed 3 Jul 2017.
20. International Organization for Standardization. ISO 11239:2012, health informatics: identification of medicinal products. Data elements and structures for the unique identification and exchange of regulated information on pharmaceutical dose forms, units of presentation, routes of administration and packaging. https://www. iso.org/standard/55032.html (2012). Accessed 3 Jul 2017.

21. International Organization for Standardization. ISO 11240:2012, health informatics: identification of medicinal products. Data elements and structures for the unique identification and exchange of units of measurement. https://www.iso.org/standard/ 55033.html (2012). Accessed 3 Jul 2017.

22. International Organization for Standardization. ISO 11615:2012, health informatics: identification of medicinal products. Data elements and structures for the unique identification and exchange of regulated medicinal product information. https:// www.iso.org/standard/55034.html (2012). Accessed 3 Jul 2017.

23. International Organization for Standardization. ISO 11616:2012, health informatics: identification of medicinal products. Data elements and structures for the unique identification and exchange of regulated pharmaceutical product information. https://www.iso. org/standard/55035.html (2012). Accessed 3 Jul 2017.

24. European Medicines Agency. Collecting high-quality information on medicines through patient registries. http://www.ema.europa. eu/ema/index.jsp?curl=pages/news_and_events/news/2015/10/ news_detail_002413.jsp\&mid=WC0b01ac058004d5c1 (2017). Accessed 3 Jul 2017.

25. Official Journal of the European Union. Commission Implementing Regulation (EU) No. 520/2012. http://eur-lex.europa.eu/ LexUriServ/LexUriServ.do?uri=OJ:L:2012:159:0005:0025:EN: PDF (2012). Accessed 3 Jul 2017.

26. Council for International Organizations of Medical Sciences. Report of the Council for International Organisations of Medical Sciences Working Group VIII Practical Aspects of Signal Detection in Pharmacovigilance (CIOMS, Geneva 2010). http:// cioms.ch/shop/product/practical-aspects-of-signal-detection-inpharmacovigilance-report-of-cioms-working-group-viii/ (2017). Accessed 3 Jul 2017.

27. European Medicines Agency. Questions and answers on signal management (Rev 2, Corr 2). http://www.ema.europa.eu/docs/ en_GB/document_library/Other/2013/09/WC500150743.pdf (2017). Accessed 3 Jul 2017.

28. European Medicines Agency. Guideline on good pharmacovigilance practices (GVP): module IX: signal management (Rev 1). Draft for public consultation. http://www.ema.europa.eu/docs/en GB/document_library/Regulatory_and_procedural_guideline/20 16/08/WC500211720.pdf (2016). Accessed 3 Jul 2017.

29. European Medicines Agency. Guideline on good pharmacovigilance practices (GVP): module VII: periodic safety update report. http://www.ema.europa.eu/docs/en_GB/document_library/Scient ific_guideline/2013/04/WC500142468.pdf (2013). Accessed 3 Jul 2017.

30. Wisniewski AFZ, Bate A, Bousquet C, et al. Good signal detection practices: evidence from IMI PROTECT. Drug Saf. 2016;39(6):469-90.

31. Pacurariu AC, Coloma PM, van Haren A, et al. A description of signals during the first 18 months of the EMA pharmacovigilance risk assessment committee. Drug Saf. 2014;37(12):1059-66.

32. European Medicines Agency. SGLT2 inhibitors (previously canagliflozin). http://www.ema.europa.eu/ema/index.jsp?curl= pages/medicines/human/referrals/SGLT2_inhibitors_(previously_ Canagliflozin)/human_referral_prac_000059.jsp\&mid=WC0b01a c05805c516f (2017). Accessed 3 Jul 2017.

33. European Medicines Agency. Thiocolchicoside-containing medicines. http://www.ema.europa.eu/ema/index.jsp?curl=pages/med icines/human/referrals/Thiocolchicoside-containing_medicines/ 
human_referral_000356.jsp\&mid=WC0b01ac05805c516f (2017). Accessed 3 Jul 2017.

34. European Medicines Agency. PRAC recommendations on safety signals. http://www.ema.europa.eu/ema/index.jsp?curl=pages/reg ulation/document_listing/document_listing_000375.jsp\&mid= WC0b01ac0580727d1c (2017). Accessed 3 Jul 2017.

35. European Medicines Agency. Periodic safety update reports, list of EU reference dates and PSUR submission. http://www.ema. europa.eu/ema/index.jsp?curl=pages/regulation/document_listing/ document_listing_000361.jsp (2017). Accessed 3 Jul 2017.

36. European Medicines Agency. Central repository for safety reports: one year to go before mandatory use. http://www.ema. europa.eu/ema/index.jsp?curl=pages/news_and_events/news/201 5/06/news_detail_002346.jsp\&mid=WC0b01ac058004d5c1 (2017). Accessed 3 Jul 2017.

37. European Medicines Agency. Referral procedures. http://www. ema.europa.eu/ema/index.jsp?curl=pages/regulation/general/ general_content_000150.jsp (2017). Accessed 3 Jul 2017.

38. European Medicines Agency. Referrals. http://www.ema.europa. eu/ema/index.jsp?curl=pages/medicines/landing/referral_search. jsp\&mid=WC0b01ac05805c516f (2017). Accessed 3 Jul 2017.

39. European Medicines Agency. Guideline on good pharmacovigilance practices (GVP): module XVI: risk minimisation measures: selection of tools and effectiveness indicators (Rev 2). http:// www.ema.europa.eu/docs/en_GB/document_library/Scientific guideline/2014/02/WC500162051.pdf (2017). Accessed 3 Jul 2017.

40. European Medicines Agency. Recommendations on medication errors. http://www.ema.europa.eu/ema/index.jsp?curl=pages/regu lation/document_listing/document_listing_000398.jsp\&mid=WC 0b01ac058098f1c0 (2017). Accessed 3 Jul 2017.

41. European Network of Centres for Pharmacoepidemiology and Pharmacovigilance. The European Union electronic register of post-authorisation studies (EU PAS register). http://www.encepp. eu/encepp_studies/indexRegister.shtml (2017). Accessed 3 Jul 2017.

42. European Medicines Agency. Public hearings. http://www.ema. europa.eu/ema/index.jsp?curl=pages/about_us/general/general_ content_001432.jsp\&mid=WC0b01ac0580a221a4 (2017). Accessed 3 Jul 2017.

43. European Medicines Agency. Guideline on good pharmacovigilance practices (GVP): module XV: safety communication. Available from: http://www.ema.europa.eu/docs/en_GB/docum ent_library/Scientific_guideline/2013/01/WC500137666.pdf (2013). Accessed 3 Jul 2017.

44. Dal Pan GJ, Arlett PR. The US Food and Drug AdministrationEuropean Medicines Agency collaboration in pharmacovigilance: common objectives and common challenges. Drug Saf. 2015;38(1):13-5.

45. European Medicines Agency. Guiding principles for the international pharmacovigilance cluster. http://www.ema.europa.eu/ docs/en_GB/document_library/Other/2014/12/WC500179390. pdf (2015). Accessed 3 Jul 2017.

46. European Medicines Agency. Letter on the working arrangement to exchange non-public information on medical products between the European Commission's DG SANTE, EMA and the WHO. http://www.ema.europa.eu/docs/en_GB/document_library/Other/ 2015/09/WC500193948.pdf (2015). Accessed 3 Jul 2017.

47. European Medicines Agency. Guideline on good pharmacovigilance practices (GVP): module I: pharmacovigilance systems and their quality systems. http://www.ema.europa.eu/docs/en_GB/ document_library/Scientific_guideline/2012/06/WC500129132. pdf (2012). Accessed 3 Jul 2017.

48. Prieto L, Spooner A, Hidalgo-Simon A, et al. Evaluation of the effectiveness of risk minimization measures. Pharmacoepidemiol Drug Saf. 2012;21(8):896-9.

49. European Medicines Agency. PRAC strategy on measuring the impact of pharmacovigilance activities. http://www.ema.europa. eu/docs/en_GB/document_library/Other/2016/01/WC500199756. pdf (2016). Accessed 3 Jul 2017.

50. European Medicines Agency. PRAC work plan: final. 2016: adopted by the Committee on 11 February 2016. http://www.ema. europa.eu/docs/en_GB/document_library/Work_programme/201 6/02/WC500202350.pdf (2016). Accessed 3 Jul 2017.

51. European Medicines Agency. Coordination of pharmacovigilance inspections. http://www.ema.europa.eu/ema/index.jsp?curl=pages/ regulation/general/general_content_000160.jsp\&mid=WC0b01ac0 58002708a (2017). Accessed 3 Jul 2017.

52. PROTECT. Welcome to the PROTECT website. http://www.imiprotect.eu/index.shtml. Accessed 3 Jul 2017.

53. European Medicines Agency. News and events. http://www.ema. europa.eu/ema/index.jsp?curl=pages/news_and_events/landing/ news_and_events.jsp\&mid=. Accessed 3 Jul 2017.

54. Heads of Medicines Agencies. EU network training centre (EUNTC): former OTSG. http://www.hma.eu/otsg.html. Accessed 3 Jul 2017.

55. SCOPE. SCOPE joint action. http://www.scopejointaction.eu/ (2017). Accessed 3 Jul 2017.

56. European network of centres for pharmacoepidemiology and pharmacovigilance. http://www.encepp.eu/index.shtml (2017). Accessed 3 Jul 2017.

57. Laporte JR. Fifty years of pharmacovigilance: medicines safety and public health. Pharmacoepidemiol Drug Saf. 2016;25(6):725-32. 